\title{
Cyclic Plasticity Based Low-Cycle Fatigue Models for Metal Structure
}

\author{
P. B. R. Dissanayake, K.R.B. Herath and S. C. Siriwardane
}

\begin{abstract}
This paper presents two phenomenological models to describe the low cycle fatigue behavior of large-scale infrastructures which are made up of non linear kinematic hardening materials. The first model basically consists of a new non linear hardening model which is suggested by making further extension to previous Armstong Fedrick model. The second model also consists of a new hardening model with incremental plasticity algorithms by providing a simple alternative to the available two surface plasticity theory. Fatigue fracture would initiate when the internal history variable reaches the threshold value and is deemed as the major concept behind the low cycle fatigue assessment. Elastoplastic behaviour of proposed models are compared with both previous models and the real material behaviour. Finally the applicability of proposed models in the vicinity of civil engineering problems is confirmed by discussing a case study.
\end{abstract}

Keywords: Elasto-plastic FEM, Low cycle fatigue, Non-linear kinematic hardening, Riveted railway bridge, Seismic design

\section{Introduction}

In the decades of 1960's and 1970's, civil engineering was dominated by design and construction of new civil infrastructure. In consideration of management of structures in terms of maintenance, it was member replacement that had a wide acceptance during this time. However, at present, many of these structures are considerably old and need maintenance, rehabilitation and replacement to keep them serviceable [1]. Detailed investigations on metal based civil infrastructure further reveal that the parameters such as age of the structure, type of use, maintenance conditions and environmental conditions influence to generate the unexpected stress concentration at a local discontinuity by producing stress-corrosion cracking $[2,3]$, release of contact ness of the mode of connection $[2,4]$, loss of sectional area of the members and change of material properties [2,4]. Thus, it is of great importance to predict the remaining service life, especially based on low cycle fatigue of existing civil infrastructures which are generally influenced by dynamic loads.

Seismic design of steel structures permits the structure to deform into the inelastic range under large ground excitations [5]. When steel members are loaded into the inelastic ranges cyclically, damage accumulations are taking place that can result in low cycle fatigue failure which is brittle in nature [6]. Some of the affected steel structures during the Great Hanshin Earthquake of January 17, 1995, illustrated that the major cause to brittle fracture was the local plastic strain concentration [7] and further it confirms the validity of above statement more realistically. In advance, it is an undesirable mode of failure in ductility-based predictions. Since only a few studies have been done in the past for seismic design applications in building and bridges under the range of low cycle fatigue, an extensive study in this area is compulsory.

Consequently, the above issues in civil engineering structures reveal the necessity of the evaluation of low cycle fatigue life for existing structures with their corresponding service life and low cycle fatigue strength evaluation (improvement of load and resistance factor) against the effect of occasional loading such as earthquake, typhoon and cyclone. These types of accidents are more severe than deliberated in the conventional limit design methods and

Eng. (Dr.) P.B. R. Dissanayake, C. Eng., MIE(SL). B.ST. Eny. (Hons; (Perndeniva). M. Eng. (Ehime). Dr. Eng. (Ehime). Se'nior Lecturer in Civil Engincering. Deparment of Civil Enpineering. University of Pe radeniya. Dr. K. R. B. Herath, B.Sc: Eng. (Hons) (Perndeniyn), M.Sc. \& Illinois.). Fh.D. (Californin.). Sentor Lecurer in Civil Engince ering. Deparment of Civil Engineering. University of Peradeniva.

S. C. Siriwurdame, B.Sr. Enss. (Homs)(Peradeniva). M.Phil (Perradeniva). 
might basically invalidate available seismic design approaches and code provisions $[7,8]$.

In low cycle fatigue problems, the behaviour of the material usually lies in the elastoplastic region. Determining stresses and plastic strains in the case of variable amplitude uniaxial loads is now a relatively easy task and there are well accepted and standard procedures (ysteresis cycles, memory rule) which are currently applied routinely in the automotive, aeronautics and aerospace industries [9]. But the engineering components of structures are generally subjected to multiaxial variable amplitude loading, which may be nonpropotional: not only may the principle axis rotate with respect to time, but also the applied loading may be of variable amplitude. Therefore, it is important to extend this technique to the multiaxial case and there is no single equivalent technique available at present for the multiaxial case [9]. However, it has been mentioned that fatigue life prediction for multiaxial random loading is an extremely large and intractable topic, due to the inherent complexities of fatigue crack initiation under a nonproportional variable amplitude nature $[9$, 10]. Therefore, the lack of multiaxial fatigue life prediction techniques has prevented the quantitative assessment of engineering structures which are subjected to more than one loading mode of a random amplitude nature.

However, as reported in literature, difficulty in the application of Basquin, Coffin-Manson and Palmgren-Miner cumulative damage law results from improper characterisation of damaging events and from not taking the interaction effect into consideration [11]. The rainflow counting method first proposed by Endo [12], was initially applied to the case of low cycle fatigue. With time, some attempts have been made to extend uniaxial fatigue concepts to variable amplitude multiaxial loading [13]. However, it is now recognized that it is erroneous to cycle count principal stress ranges or von Mises equivalent stress ranges using conventional cycle counting methods, as the negative half cycles would be folded over. In the case of fully reversed uniaxial loading, the counted range is only half the actual range[14]. One of the best known low cycle fatigue design codes that address nonpropotional loading problems is the ASME boiler and pressure vessel code, which offered a way to assimilate stresses of a simple nonpropotional loading cycle into a scalar quantity to enter the fatigue curves. Since history reveals that these models are basically useful for prediction of low cycle fatigue life in a macroscopic sense [15], it is strongly needed to consider microscopic sense of failure and these techniques are collectively designated as local strain approach or state variable approach to fatigue life prediction. Consistent with the current advances in computing technology and the wealth of micro and nano technology of material science, it was revealed that the successive application of plastic strain is the principle cause of fatigue crack initiation[16,18]. However, with reference to the literature, even though the predictions from this method were found to be in the field of mechanical engineering, applications to civil engineering structures were not found to be in the low cycle fatigue regime.

Consequently, this paper presents two phenomenological fatigue models which are generated using the two fundamental approaches in the cyclic plasticity theory, with new ideas appended to the classical ideas for non-linear kinematics hardening materials. The concept behind the methodology is the determination of the internal state variable of material at the place where the stresses are most severe, when it is subjected to multiaxial variable amplitude loading. Fatigue life of the component is assumed to be over when the internal state variables reach the threshold values of the material $[18,20]$.

\section{Rate independent cyclic plasticity theory}

This section briefly reviews some of the important concepts of the rate independent cyclic plasticity. This provides the major basis for the present fatigue approaches. The three basic elements of an incremental plasticity theory are yield criteria, a flow rule and a hardening rule [21].

A vast number of models of varying levels of sophistication have been developed to model the elasto-plastic behavior of metals under different loading conditions. Major classes of these models include the multisurface models of Mroz[22] with related two surface theories [21, 
23-26] and the non-linear kinematic model of Armstrong and Frederick [27]. The major difference among these different plasticity models available is the hardening rules, which describe the movement of the yield surface corresponding to the kinematic hardening and the change in the size of the yield surface corresponding to the isotropic hardening. The yield surface separates the elastic and plastic response regions, which is characterized by its centre and radius represented by the backstress and inner yield strength, respectively. The crucial element of these theories is the so-called hardening rule, a prescription to specify the movement of the yield surfaces in stress space.

The available non-linear kinematic hardening models for multaxial cyclic plasticity still produce slightly incorrect predictions to their behavior when it reaches high values of total strains or effective plastic strains. Even though the multisurface model of Mroz[22] and related two surface theories [23] seems to be the most frequently used approach to solve the problem to some extent, only simple constitutive models are implemented in most of the standard finite element (FE) programmes [28]. This causes inaccurate predictions in some materials or structures in the cases of ratcheting (cycle by cycle accumulation of permanent deformation) behavioral analysis, low cycle fatigue life estimation and etc $[27,28]$. Further, it is obvious that the determination of hardening parameters of two surface type models generally depend on complex series of laboratory experiments of corresponding material [29]. Thus, it tends to reduce the application of the two surface theory in elasto-plastic applications especially in the civil engineering field.

Consequently, this paper proposes a fatigue model (Fatigue model I) mainly consisting of Amstrong Fedrick type cyclic elasto-plastic theory and a second model (Fatigue model II) which includes the two surface cyclic plasticity theory. The respective elasto-plastic related hardening models are newly proposed by the authors in this paper and the applicability of the two proposed constitutive relations are not limited to low cycle fatigue estimation but also can be used in other non linear elasto-plastic analysis of kinematic hardening materials.

\section{Fatigue model I}

The fatigue model was developed to predict the low cycle behavior of the non-linear kinematic hardeing material using a new elasto-plastic model which is proposed by the authors. The elasto-plastic model consists of a new hardening rule and it has been suggested by making a little extension of previous Armstrong - Fredrick rule [27]. The new term is a function of the stress increment and it is used to define the direction of yield surface. The concept behind the low cycle fatigue assessment is a determination of the critical history variable of the material (or structure) when it is subjected to a fluctuating load at the place where the stresses are most severe. The fatigue fracture would initiate at such places when the internal history variable reaches the threshold value which should be determined by a series of experiments [18-20]. Considering that metals mostly used in structural engineering have an appreciable strain-hardening rate, effective plastic strain is taken as the critical internal state variable in this model.

\subsection{Proposed hardening model}

In plasticity theory [21], for a material exhibiting the kinemetic hardening behavior, the von Misses yield criteria is defined as,

$\phi=\left(\sigma_{i j}^{\prime}-\alpha_{i j}^{\prime}\right)\left(\sigma_{i j}^{\prime}-\alpha_{i j}^{\prime}\right)-\frac{2}{3} \sigma_{0}^{2}=0$

where $\phi=$ the yield function; $\sigma t_{i j}^{\prime}=$ the deviatoric stress tensor; $\sigma t_{i j}^{\prime}=$ the deviatoric component of back stress tensor; and $\sigma_{0}=$ the initial yield stress in uniaxial tension.

When the plastic flow is assumed to be associated, the incremental plastic strain is found for an increase of load in the plastic region from the Drucker's normality condition and it is mathematically described as,

$d \varepsilon_{i j}^{p}=d \lambda\left(\frac{\partial \phi}{\partial \sigma_{i j}^{\prime}}\right)$

where $d \lambda=$ the plastic multiplier; and $d \varepsilon_{i j}=$ the incremental plastic strain.

After the elastic limit is reached, the state of stress lies on the yield surface and, here, hardening follows kinematic hardening behaviour. The hardening rule is proposed as below by making a little extension of previous Armstrong-Fredrick rule. 
$d \alpha_{i j}=\frac{2}{3} c d \varepsilon_{i j}^{p}-\gamma \alpha_{i j} d \bar{\epsilon}^{p}+\beta d \sigma_{i j}$

where $c, \gamma, \beta=$ hardening constants, which can be determined by the uniaxial experimental results of particular material; and $d \alpha_{i j}=$ the incremental back stress tensor which perfectly describes the manner of yield surface translation in stress space.

The first term $c d \varepsilon^{p}{ }^{\prime}$, describes the linear behaviour of yield surface translation with the change of plastic strain and its direction is perpendicular to the yield surface. The second term $-\gamma \alpha_{i j} d \varepsilon^{p}$, consists of the back stress vector. Since the back stress $\alpha_{i j}$ increases with the increment of plastic loading, the second term $\gamma \alpha_{i} d E^{-p}$ also changes in both magnitude and direction. As a consequence, the present yield surface translation manner exhibits non-linear behaviour. The new term $\beta d \sigma_{i j}$ is increasing with the plastic loading and, finally, it contributes to increase the effect of non-linear behaviour more, especially in large strain range.

For loading increment in the plastic region the effective plastic strain is defined as,

$d \bar{\varepsilon}^{p}=\sqrt{2 / 3 d \varepsilon_{i j}^{p} d \varepsilon_{i j}^{p}}$

where $d \varepsilon^{p}=$ the incremental effective plastic strain; and $d \varepsilon_{i j}^{p}=$ the incremental plastic strain tensor.

Further manipulation of Eqs. (2) and (4) with Eq. (1), the effective plastic strain is eliminated as,

$d \bar{\varepsilon}^{p}=4 / 3 d \lambda \sigma_{0}$

During this plastic loading process the accumulated effective plastic strain is updated to obtain the current state of effective plastic strain.

$\left(\bar{\varepsilon}^{p}\right)_{\text {new }}=\left(\bar{\varepsilon}^{p}\right)_{\text {old }}$

where $\left(E^{p}\right)_{\text {New }}=$ the current effective plastic strain; and $\left(E^{\circ}\right)_{\text {old }}=$ the previous step effective plastic strain.

For no change in plastic strain for a load increment in the elastic region the current effective plastic strain is decomposed as,

$\left(\bar{\varepsilon}^{p}\right)_{\text {new }}=\left(\bar{\varepsilon}^{p}\right)_{\text {old }}$
The updated effective plastic strain should be checked with the threshold value at failure which is determined by the series of experiments of particular material. When the updated effective plastic strain reaches the threshold value of the material, the low cycle fatigue life is considered to be over.

\section{2 The elasto-plastic tangent stiffness}

Finite Element (FE) formulation of the elastoplastic stiffness matrix for incremental loading is described below.

The function $\phi$ is such that the material is elastic for $\phi \leq 0$ and $\left(\partial \phi / \partial \sigma_{i j}^{\prime}\right) d \sigma_{i j}^{\prime}<0$. In this case the material is described by,

$d \sigma_{l j}=E_{\mid j k} d \varepsilon_{k l}^{e}$

where $E_{i j|k|}=$ material elastic stiffness matrix; and $d \varepsilon_{k t}{ }_{k}=$ the incremental elastic component of total strain.

If $\phi=0$ and $\left(\partial \phi / \partial \sigma_{i j}^{\prime}\right) d \sigma_{i j}^{\prime} \geq 0$ then the process is said to be plastic and the incremental stress is decomposed as,

$d \sigma_{i j}=E_{i j t}\left(d \varepsilon_{k l}-d \lambda\left(\frac{\partial \phi}{\partial \sigma_{k l}^{\prime}}\right)\right)$

For continued yielding beyond the initial yielding, the consistency condition in plasticity defines,

$d \phi=\frac{\partial \phi}{\partial \sigma_{i j}} d \sigma_{i j}+\frac{\partial \phi}{\partial \alpha_{i j}} d \alpha_{i j}=0$

Considering the relations between deviatoric stress to total stress, Eq. (10) can be simplified as,

$\cdot\left(\frac{\partial \phi}{\partial \sigma_{i j}^{\prime}}\right)\left(d \sigma_{i j}-d \alpha_{i j}\right)=0$

This condition is used together with definitions and new hardening rule in Eq. (11) to obtain plastic multiplier,

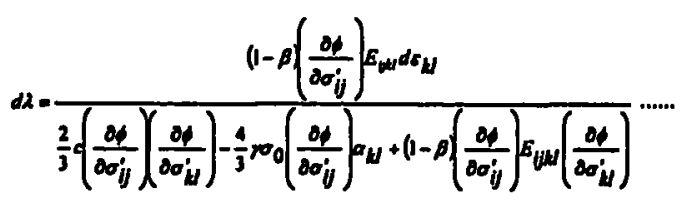

and further manipulation of Eq. (9) the material elasto-plastic stiffness tensor is deduced as,

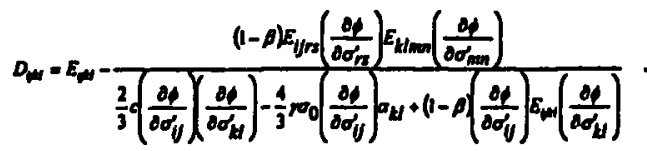


Finally the obtained $D_{i j k l}$ is used to formulate global elasto-plastic stiffness matrix for the whole structure in FE programme code.

\section{3 Determination of hardening constants}

Determination of hardening constants associated with any proposed material model is one of the most challenging issue for researchers in order to obtain better representation of their hardening behavior. The identification procedure for the material constants involved in the described back stress evolution equation is based on available experimental results. If limited data are available, $c, \gamma$, and $\beta$ can be based on the stress- strain data obtained from the half cycle of the uniaxial tension or compression experiments. The details of the procedure to determine the material constants are outlined below.

From the uniaxial specialization of the Eqs. (11) and (4),

$d \alpha=d \sigma$ and $d \varepsilon_{p}=d \varepsilon_{p}$

Where the $\sigma, \alpha, \varepsilon_{\eta}$, are the corresponding uniaxial stress, back stress and plastic strain respectively.

Utilizing Eq. (14), Eq. (3) is rewritten as to obtain the view of uniaxial behavior,

$d \alpha=\frac{2}{3} c d \varepsilon^{p}-\gamma \alpha d \varepsilon^{p}+\beta d \sigma$

Integrating the above expression over a half cycle of the stress strain data yields the following expression,

$\alpha=\frac{2 c}{3 \gamma}+\left(\alpha_{0}-\frac{2 c}{3 \dot{\gamma}}\right) \exp \left[\frac{-\gamma}{1-\beta}\left(\varepsilon^{p}-\varepsilon_{0}^{p}\right)\right]$

and the $\left(\varepsilon_{0}^{p}, \alpha_{0}\right)$ results from the previous flow.

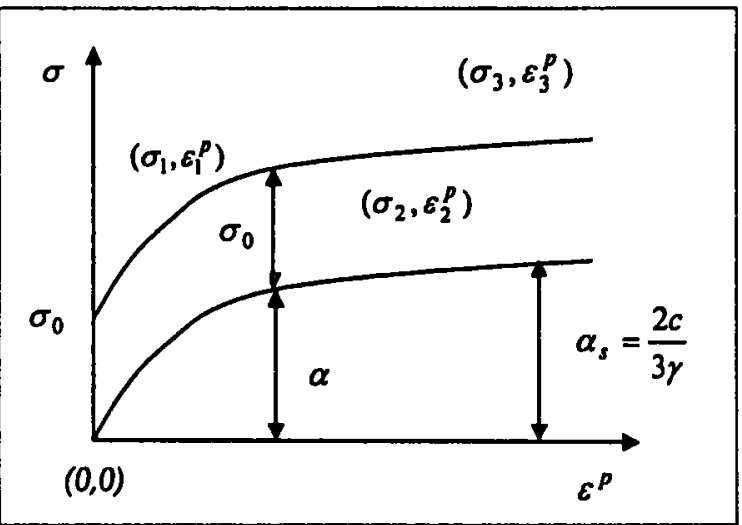

Fig. 1. Half cycle of uniaxial stress-strain data representing the non-linear hardening.
Using the finite set of points in the uniaxial back stress-plastic strain curve (Fig. 1), one can approximate the curve in the form of Eq. (16). Close to the saturation point of hardening process, the hardening increments tend to zero and hence the saturation back stress,

$\alpha_{s}=\frac{2 c}{3 \gamma}$

hence, Eq. (16) is simplified as,

$\ln \left[\frac{\alpha_{s}-\alpha_{0}}{\alpha_{s}-\alpha}\right]=\frac{\gamma}{1-\beta}\left(\varepsilon^{p}-\varepsilon_{0}^{p}\right)$

The Eq. (18) is of the form of $y=a x$ with,

$y=\ln \left[\frac{\alpha_{s}-\alpha_{o}}{\alpha_{s}-\alpha}\right], a=\frac{\gamma}{1-\beta}$, and $x=\varepsilon^{p}-\varepsilon_{0}^{p}$

which is the equation of a straight line. The application of least squares fit of the transformed variables in the forgoing form the $c$ and $\gamma$ can be obtained as,

$c=\frac{3}{2} \alpha_{s} a(1-\beta)$ and $\gamma=a(1-\beta)$

Then the value of $\beta$ is determined by minimizing the squares of errors as Eq. (21).

$e^{2}=\sum_{i=1}^{n}(\bar{\alpha}-\alpha)^{2}$

where, $e=$ the error, $\bar{\alpha}=$ back stress value from the actual data at the $n$ data points; and $\alpha=$ back stress value illustrates as a function of $\beta$. The value of the $\beta$ is determined when the error is smallest.

Finally the obtained constants are subjected to trial and error investigation with the experimental data until it provides the best fit to the real behavior of the material and hence determine the final $c, \gamma, \beta$ values for the corresponding material.

\section{Fatigue model II}

The second fatigue model is also developed to predict the low cycle behavior of the non-linear kinematic hardeing materials using a new elasto-plastic model which is proposed by the author. The elasto-plastic model consists of a new hardening rule and it has been suggested to furnish a simple alternative to existing two surface models [21, 23-26]. The concept behind the low cycle fatigue assessment is also the same 
as Fatigue model I, (chapter 3) and the effective plastic strain is taken as the critical internal state variable of the material in this model also.

\subsection{Proposed hardening model}

In plasticity theory[21], for a material exhibiting the kinemetic hardening behavior as shown in Fig. (2), the von Misses yield criteria is defined as,

$\phi=\left(\sigma_{i j}^{\prime}-\alpha_{i j}^{\prime}\right)\left(\sigma_{i j}^{\prime}-\alpha_{i j}^{\prime}\right)-\frac{2}{3} \sigma_{0}^{2}=0$

$\phi=S_{i j} S_{i j}-\frac{2}{3} \sigma_{0}^{2}=0$

where $\phi=$ the yield function; $\sigma_{i j}^{\prime}=$ the deviatoric stress tensor $\sigma_{i j}^{\prime}=$ the deviatoric component of back stress (residual stress) tensor which controls the kinematics hardening behaviour; $\sigma_{0}=$ the initial yield stress in uniaxial tension; and $S_{i j}=\sigma^{\prime} i j-\alpha^{\prime} i j$.

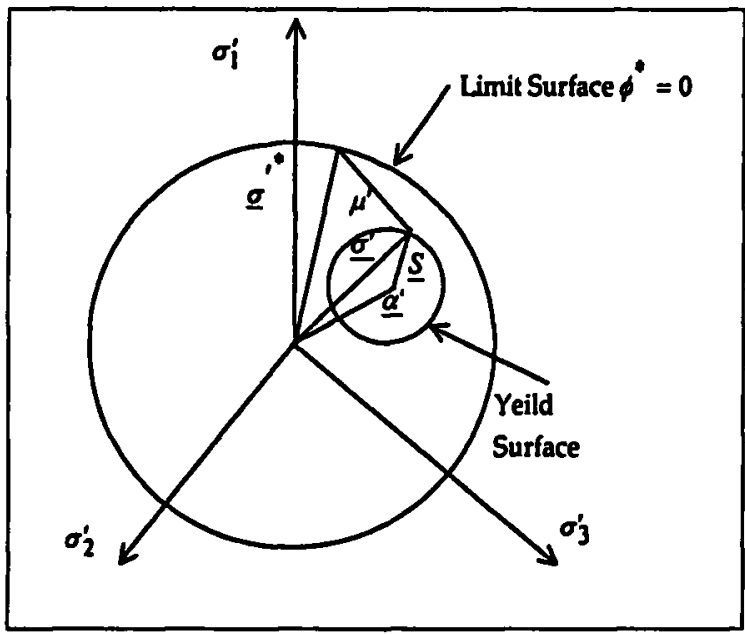

Fig. 2. Schematic representation of limit and yield surfaces in $\pi$-plane.

When the plastic flow is assumed to be associated, the incremental plastic deviatoric strain is found for an increase of load in the plastic region from the Drucker's normality condition and it is mathematically described as,

$d \varepsilon_{j j}^{\prime p}=d \lambda\left(\frac{\partial \phi}{\partial \sigma_{i j}^{\prime}}\right)$

where $d \lambda=$ the plastic multiplier; and $d \varepsilon_{i j}^{p}=$ the deviatoric component of incremental plastic strain.

In two surface plasticity theory [21, 23-26], the hardening of the loading surface depends upon the distance vector from the current stress state to the limit surface. The schematic diagram of both loading and limit surfaces in $\pi$-plane are shown in Fig. 2. When the limit surface does not move in the stress space, it can be mathematically defined as,

$\phi^{*}=\sigma_{i j}^{\prime *} \sigma_{i j}^{\prime *}-\frac{2}{3} \sigma_{u t s}^{2}=0$

where $\phi^{*}=$ the limit function; $\sigma_{u / s}=$ the ultimate tensile strength of the material; $\sigma_{i j}=$ the stress tensor corresponding to ultimate strength of the material; and $\sigma^{*}{ }_{i j}=$ the deviatoric component of stress tensor corresponding to ultimate strength of the material.

In two surface plasticity model, the deviatoric stress vector corresponding to the ultimate strength of limit surface is parallel to the radial stress vector in loading surface as shown in Fig. 2. Thus the loading surface touches the limit surface at similar points on two surfaces as in Fig. 2. The distance between these two points is the vector $\mu_{i j}^{\prime}$ and for this model with two surfaces, the hardening rate is proportional to the distance between similar points in two surfaces. When the stress state is remote from the limit surface the system is very stiff. When the stress state is near the limit surface, the system essentially adopts the stiffness or hardening modulus of the limit surface. Thus the system varies smoothly from high to low stiffness during a plastic loading process. Therefore, for a non-linear kinematic hardening behavior of a material, the hardening rule is defined as,

$d \alpha_{i j}^{\prime}=A d \lambda \mu_{i j}^{\prime}$

$\mu_{i j}^{\prime}=\left(\sigma_{i j}^{\prime *}-\sigma_{i j}^{\prime}\right)$

where $\mu_{i j}^{\prime}=$ the distance vector; and $\mathrm{A}=$ the hardening parameter which simplifies to a constant in kinematic hardening.

For loading increment in the plastic, a definition for the effective plastic strain is the mean of the deviatoric plastic strain, as described in the Eq. (4)-(7) in the fatigue model I (chapter 3), and low cycle fatigue life prediction concept is also a determination of effective plastic strain of the particular material as described in the fatigue model I.

\section{2 The elasto-plastic tangent stiffness}

Finite Element formulation of the elasto-plastic stiffness matrix for incremental loading is described below. 
The function $\phi$ is such that the material is elastic for $\phi \leq 0$ and $\left(\partial \phi / \partial \phi_{i j}^{\prime}\right) d \sigma_{i j}^{\prime}<0$. In this case material is described by,

$d \sigma_{i j}^{\prime}=E_{i j k} d \varepsilon_{k l}^{\prime e}$

where $E_{j i k t}=$ material elastic stiffness matrix; and $\mathrm{d}^{\prime{ }_{k l}^{\prime}}=$ the incremental elastic component of total deviatoric strain.

If $\phi=0$ and $\left(\partial \phi \partial \phi_{i j}^{\prime}\right) d \sigma_{i j}^{\prime} \geq 0$ then the process is said to be plastic and the incremental deviatoric stress is decomposed as,

$d \sigma_{i j}^{\prime}=E_{i j k}\left(d \varepsilon_{k l}^{\prime}-d \lambda\left(\frac{\partial \phi}{\partial \sigma_{k l}^{\prime}}\right)\right)$

For continued yielding beyond the initial yielding, the consistency condition in plasticity defines,

$d \phi=\frac{\partial \phi}{\partial \sigma_{i j}^{\prime}} d \sigma_{i j}^{\prime}+\frac{\partial \phi}{\partial \alpha_{i j}^{\prime}} d \alpha_{i j}^{\prime}=0$

Considering the relations between deviatoric stress to deviatoric back stress, Eq. (30) can be simplified as,

$\left(\frac{\partial \phi}{\partial \sigma_{i j}^{\prime}}\right)\left(d \sigma_{i j}^{\prime}-d \alpha_{i j}^{\prime}\right)=0$

This condition is used together with definitions and new hardening rule in Eq. (31) to obtain plastic multiplier,

$$
d \lambda=\frac{\left(\frac{\partial \phi}{\partial \sigma_{i j}^{\prime}}\right) E_{i j k l} d \varepsilon_{k l}}{A\left(\frac{\partial \phi}{\partial \sigma_{i j}^{\prime}}\right)\left(\sigma_{i j}^{\prime *}-\sigma_{k l}^{\prime}\right)+\left(\frac{\partial \phi}{\partial \sigma_{i j}^{\prime}}\right) E_{i j k l}\left(\frac{\partial \phi}{\partial \sigma_{k l}^{\prime}}\right)}
$$

and further manipulation of Eq. (29) the material elasto-plastic stiffness tensor deduced as,

$$
D_{i j t}=E_{j j t}-\frac{E_{i j r s}\left(\frac{\partial \phi}{\partial \sigma_{r s}^{\prime}}\right) E_{k l m n}\left(\frac{\partial \phi}{\partial \sigma_{m n}^{\prime}}\right)}{A\left(\frac{\partial \phi}{\partial \sigma_{i j}^{\prime}}\right)\left(\sigma_{k l}^{*}-\sigma_{k l}^{\prime}\right)+\left(\frac{\partial \phi}{\partial \sigma_{i j}^{\prime}}\right) E_{i k l}\left(\frac{\partial \phi}{\partial \sigma_{k l}^{\prime}}\right)}
$$

Finally the obtained Dijkl is used to formulate global elasto-plastic stiffness matrix for the whole structure in FEM programme code with utilizing deviatoric stress and strain state.

\section{3 Determination of hardening constant}

The theory presented in the proceeding section has a single constant $A$, which is related to the particular material. Subjecting to the fatigue model to uniaxial stress specialization and hence comparing uniaxial tensile test up to the failure of the material is used to determine the unknown constant $\mathrm{A}$ in this fatigue model.

When examined for the case of uniaxial stress under a nonreversing load history, the Eq. (29) can be specialized as,

$d \sigma_{i j}^{\prime}=d \alpha_{i j}^{\prime}$

Further manipulation of Eq. (26) and (34), the incremental stress tensor is simplified as,

$d \sigma_{i j}^{\prime}=A d \lambda\left(\sigma_{i j}^{\prime *}-\sigma_{i j}^{\prime}\right)$

The elimination of principal stress and respective deviatoric components at considered point for uniaxial tension the incremental uniaxial stress state is resulted as,

$d \sigma=\operatorname{Ad\lambda }\left(\sigma^{*}-\sigma\right)$

where $\sigma=$ the value of uniaxial stress at a particular point of the material; and $\sigma^{*}=$ the ultimate tensile strength of the material.

Requirement of approach through stress strain gradient of the material and application of total strain increment with respective elastic and plastic strain components for uniaxial nonreversing loading operations, the Eq. (36) is eliminated as,

$d \varepsilon^{p}+\frac{d \sigma}{E}=\frac{A d \lambda\left(\sigma^{*}-\sigma\right)}{\left|\frac{d \sigma}{d \varepsilon}\right|}$

Based on the definition of effective plastic strain in plasticity, the incremental plastic strain along the uniaxial loading direction is resulted as,

$d \varepsilon^{p}=d \bar{\varepsilon}^{-p}=\frac{4}{3} d \lambda \sigma_{0}$

In the case of plastic loading operation, the plastic multiplier is to be a nonzero value $\{\mathrm{d} \lambda \neq 0)$ and further derivation of Eq. (37) and (38), the plastic parameter $A$ is determined as,

$A=\left[\frac{4 / 3\left|\frac{d \sigma}{d \varepsilon}\right| \sigma_{0}}{\sigma^{*}-\sigma}\right] \frac{1}{\left[1-1 / E\left|\frac{d \sigma}{d \varepsilon}\right|\right]}$ 
With respect to the different points selected in the experimental stress/strain diagram, different values of $A$ could be determined and a value which tends to make the best fit to the actual stress/strain behaviour of material is selected as the best value for $A$.

\section{Finite Element formulation}

The in-house FEM code is modified to utilize proposed hardening models separately. Since cyclic inelastic analysis often leads to extensive computations, it is equally important to develop accurate, robust and efficient computational algorithm. Algorithm to simulate the smooth stress state on set of yielding and elastic to plastic loading/unloading criterion in incremental plastic analysis[30] and a semiimplicit algorithm[31] which is utilized to overcome the yield surface drift are discussed below.

\subsection{Elastic to plastic loading/unloading criterion}

The algorithm is developed for the integration of incremental constitutive equations entering the cyclic plasticity models. An incremental set of equations is composed of an elastic predictor and plastic corrector. In the elastic predictor step, the incremental strain vector, $d \varepsilon_{n+r}$ is imposed while keeping the plastic internal variables constant and equal to their previous $n^{\text {th }}$ step value. This leads to an elastic trail stress read as,

$\sigma_{n+1}^{e}=E\left(\varepsilon_{n+1}-\varepsilon_{n}^{p}\right)$

where the $E$ is material elastic stiffness matrix. It is obvious that the elastic trail state is inadmissible if and only if plastic flow occurs during the $(n+1)^{\text {th }}$ step, and thus for elastic behaviour, the elastic trail state coincides with the real state at $(n+1)$ th step. Hence, the discrete loading / unloading criterion can be rewritten in terms of the elastic trail state as,

$\phi\left(\sigma_{n+1}^{e}, \alpha_{n}\right)=\left\{\begin{array}{l}\leq 0 \Rightarrow \text { elasticstate } \\ >0 \Rightarrow \text { plasticstate }\end{array}\right.$

Despite the above, descriptions are limited to total stress and strain notations and the correspondences are applicable to deviatoric sense, also in a similar manner. To simulate the smooth stress state at a set of yielding, the linear interpolation based simple approximation is utilized[32].

\section{2 Simple semi-implicit correction algorithm}

This algorithm corrects implicitly the updated quantities in order to avoid the yield surface drift. The discrete consistency method is used in the proposed method such that the final state of yield condition, after the end of $(n+1)^{\text {th }}$ step, is given by,

$\phi_{n+1}\left(\sigma_{i j}^{\prime}+d \sigma_{i j}^{\prime}, \alpha_{i j}^{\prime}+d \alpha_{i j}^{\prime}\right)=\phi_{n}\left(\sigma_{i j}^{\prime}, \alpha_{i j}^{\prime}\right)+d \phi=0 \ldots \ldots$

where $d$ symbol indicates the difference between the values of a variable at the end of the $(n+1)^{\text {th }}$ step and the $n^{\text {th }}$ step. The $d \phi$ contains the incremental terms yielding to a polynomial equation in $d \lambda$ and is called the plastic multiplier which is needed for drift correction in $(n+1)^{\text {th }}$ load increment. Finally it is simplified as,

$\phi_{n+1}(d \lambda)=0$

when considering the variables which, related to the current increment, are constants. Since the simplified mode of the equation consists only of a single variable, the local Newton-Raphson method is suggested to solve the correct value for $d \lambda$ for each successive iteration. The $d \lambda$ which is calculated directly after the present of strain increment of FE calculation is considerd as the initial guess for Newton- Raphson iteration.

\section{Verification of proposed models}

In favor of verification, the models' predicted behaviours were compared with previously proposed models and the experimental behaviours of two materials such as $316 \mathrm{~L}$ stainless steel material[26] and wrought iron[17] or both uniaxial monotonic and cyclic loading cases. To exhibit the significance and the applicability of the proposed models in the usage of real elasto-plastic applications, two numerical examples are presented at the end of this chapter.

\subsection{Model comparison with experimental behaviors}

The modified in-house FEM code is used to simulate the uniaxial monotonic tensile loading of 316L stainless steel and wrought iron, which has nearly similar properties of mild steel (SS 400 ) and has been used in construction before introducing steel to the field. To strengthen the validity of the proposed model, previous model representation behavior of same material is also compared as shown in Fig. 3 and 4 respectively. 


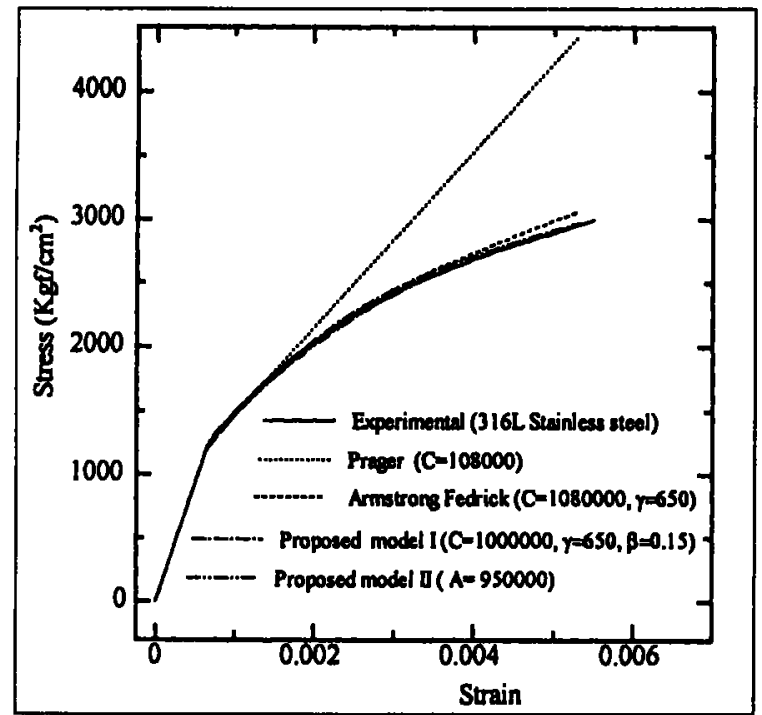

Fig. 3. Comparison of uniaxial tensile loading behavior of stainless steel $316 L$.

The results reveal that the models' prediction has better agreement with real behavior of the material than previous models and it exhibits more non-linearity than others. Particularly the material is hardening in large strain ranges (Fig. 4) and the correlation between experiment and both proposed models are well confirmed.

The satinlees steel 316L material response in a stress- controlled environment is obtained using the modified FEM code. The uniaxial stress is made to oscillate between $\pm 2300 \mathrm{kgf} / \mathrm{cm}^{2}$ and the corresponding response is plotted in Fig. 5.

The comparison revealed that even though the proposed model I has a good agreement with real behavior in cyclic range of the material than all other models, the response of model II is stabilized after a single cycle, which is a necessary requirement of the non-linear kinematic hardening materials in the case of symmetrical cyclic loading, particularly in large strain amplitude.

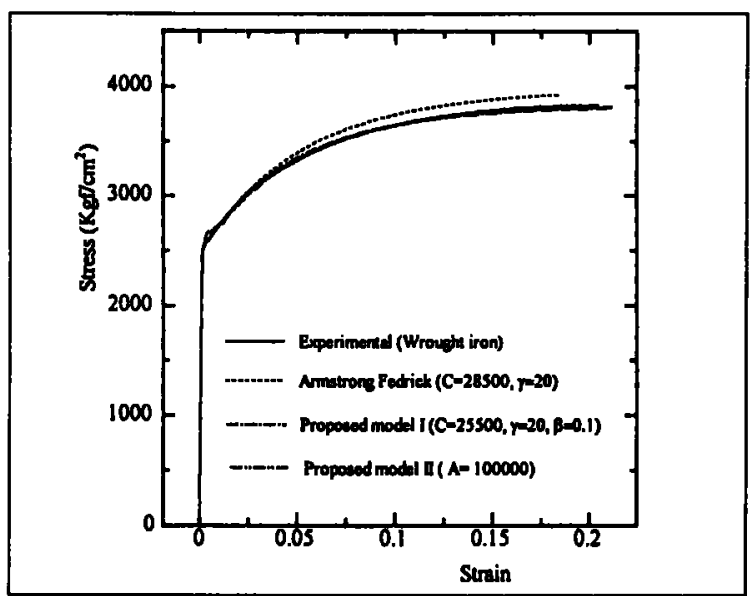

Fig. 4. Comparison of uniaxial tensile loading behavior of wrought iron material.
The model was utilized to correlate the material behavior of wrought iron under uniaxial proportional loading behavior with a single cycle of tension-compression loading in a displacement controlled environment as shown in Fig. 6.

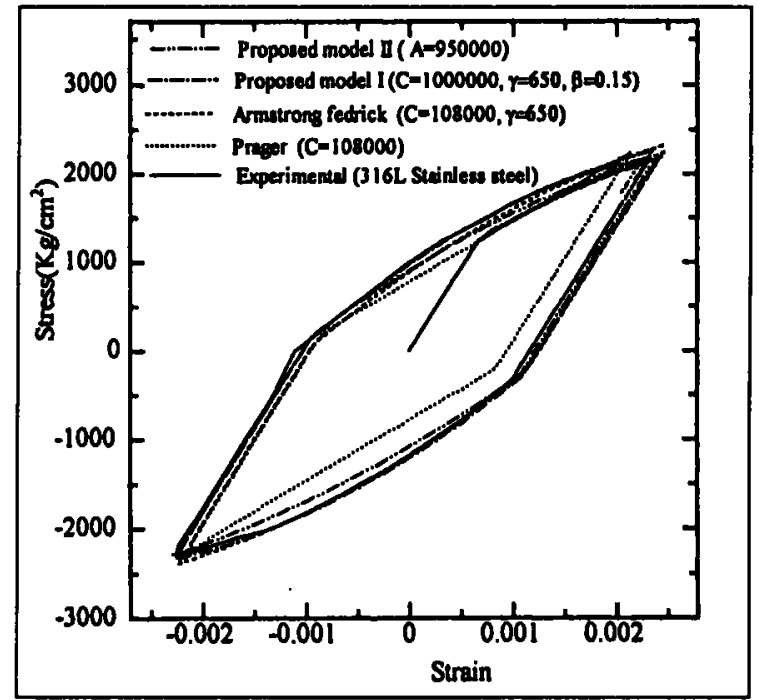

Fig. 5. Comparison of uniaxial cyclic loading behavior of stainless steel $316 L$.

The comparison illustrates that the models correlate reasonably with the experimental behaviour of the material under reverse and cyclic loading. The deviation of the models from the experimental behaviour are mainly due to the small amount of isotropic hardening shown by the wrought iron material which is not captured in the proposed models.

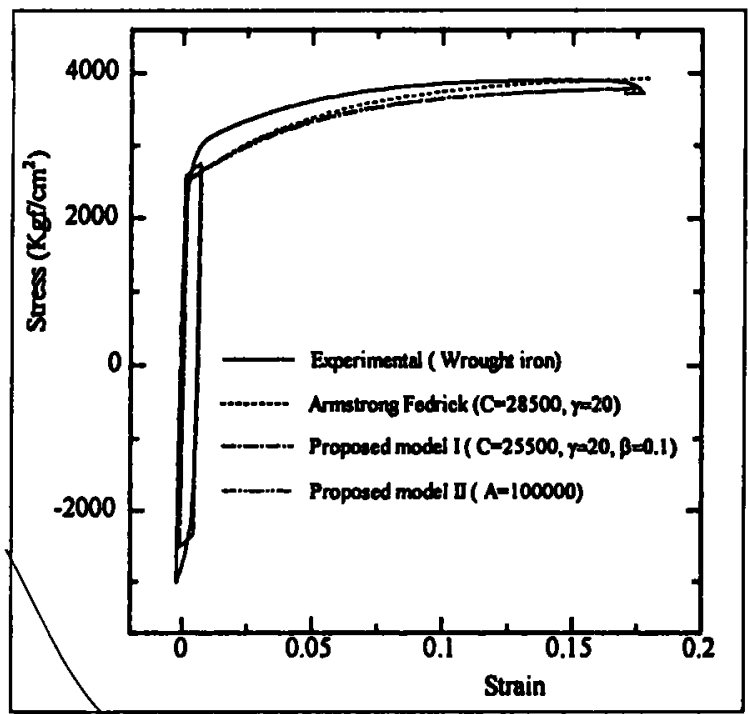

Fig. 6. Comparison of uniaxial cyclic loading behavior of wrought iron material.

With respect to all these comparisons of different loading operations it can be said that the models prediction has a reasonable agreement with the 
actual uniaxial behaviours of the material. Hence, it is confirmed the validity of proposed models in usage of elasto-plastic analysis in structures which are constructed by kinematic hardening materials.

\subsection{Significance of proposed models}

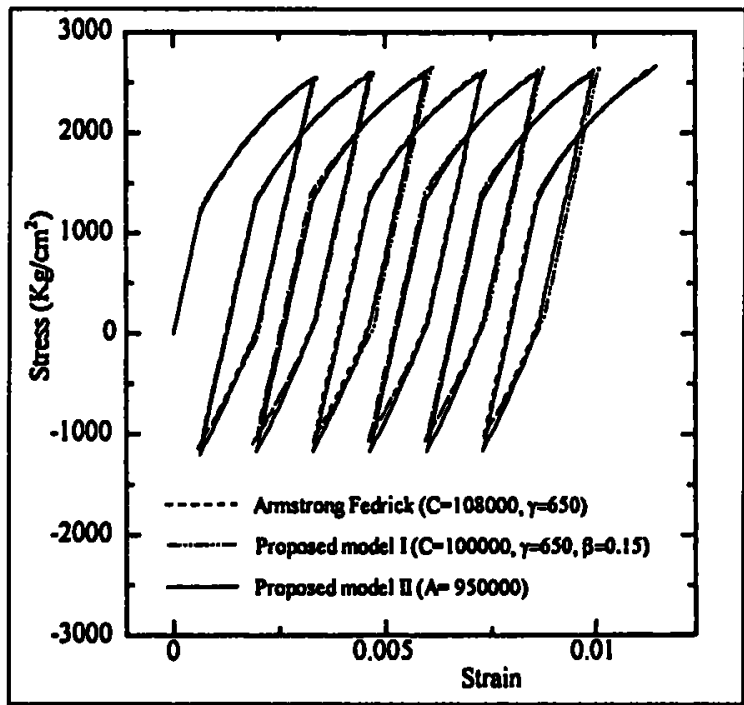

Fig. 7. Comparison of uniaxial ratcheting behavior of stainless steel $316 \mathrm{~L}$.

Fig. 7 illustrates the stress-strain curves of a stainlees steel 316L bar under uniaxial stress cycling with non-zero mean stress (ratcheting load) with a cycle number 6 . It is seen that the ratcheting can be predicted with the present models and the behavior is also a little different from the previous model. In advance, Fig. 8 exhibits the corresponding behaviour of effective stress versus effective plastic strain for the same ratcheting operation and it reveals that there is a

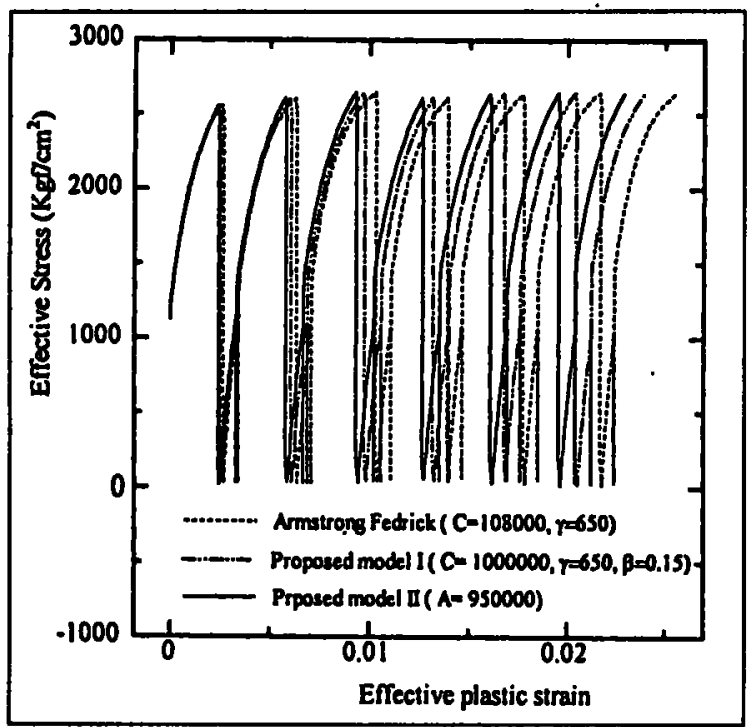

Fig .8. Comparison of effective stress vs effective strain behavior for ratcheting load of stainless steel $316 \mathrm{~L}$. significant difference among the Armstrong Fedrick models as well as between proposed models also. Since the effective plastic strain is a critical history variable of considered models, it is possible to recommend that the selection of a proper elasto-plastic model is required for failure estimation of infrastructures.

Since all above applications are limited to the uniaxial stress states with uniform elastic to plastic strain transmission, the comparison with multiaxial elasto-plastic application is presented as the next step.

A stainless steel 316L riveted plate member which is subjected to a constant amplitude uniform cyclic load in the range of \pm 9 Tons (Fig. 9-(a)), applied to the far end from the rivets, is considered for elastoplastic analysis. The geometry consists of $180^{\star} 10$ $\mathrm{mm}$ plate cross section with two symmetrically located rivet holes $60 \mathrm{~mm}$ apart with $20 \mathrm{~mm}$ diameter as shown in Fig. 9-(a). By considering the symmetricity of the geometry, loading as well as boundary condition of the member, half of the member with one rivet hole was subjected to analysis using the modified in-house FEM code. The nine node isoperimetric shell element was used for the FE mesh as shown in Fig.10-(a). To simulate the unilateral contacts between rivet and plate, a fully bonding restraint condition was employed.

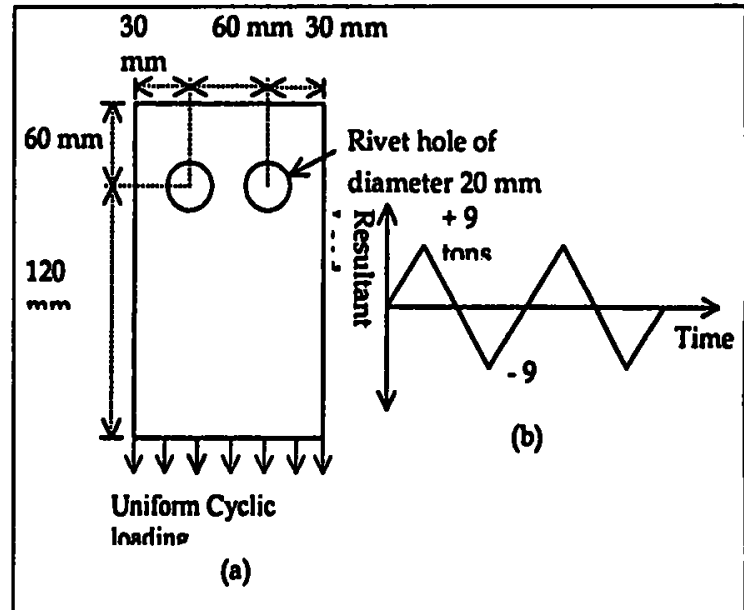

Fig. 9. Stainless steel $316 \mathrm{~L}$ riveted plate member (a). Geometric details (b). Load time history

The relevant von Misses stress contour which assists to identify the highly stressed location of the member for plastic loading is as illustrated in Fig. 10-(b). The obtained effective stress versus effective plastic strain variation for single complete cycle at critically stressed location is plotted in Fig.11. The corresponding results 


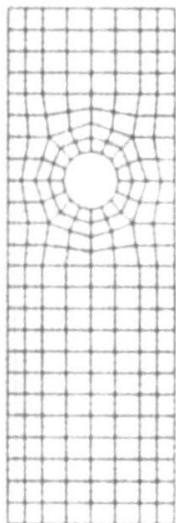

(a)

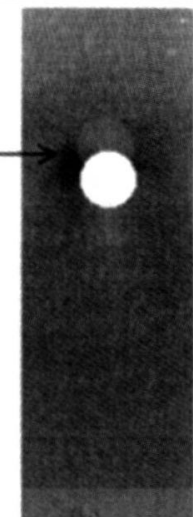

(b)

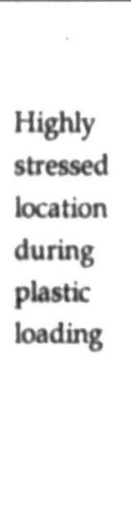

Fig. 10. FE analysis results (a). FEM mesh (b). von Misses stress distribution at plastic loading. (Darkness shows the higher magnitude of stress)

reveal that there is a major difference between these three models and related failure behavior also significantly deviates from each other when we consider the accumulated effective plastic strain as the critical variable of the material. Further, it emphasizes the significance of the proper hardening model in the vicinity of failure analysis

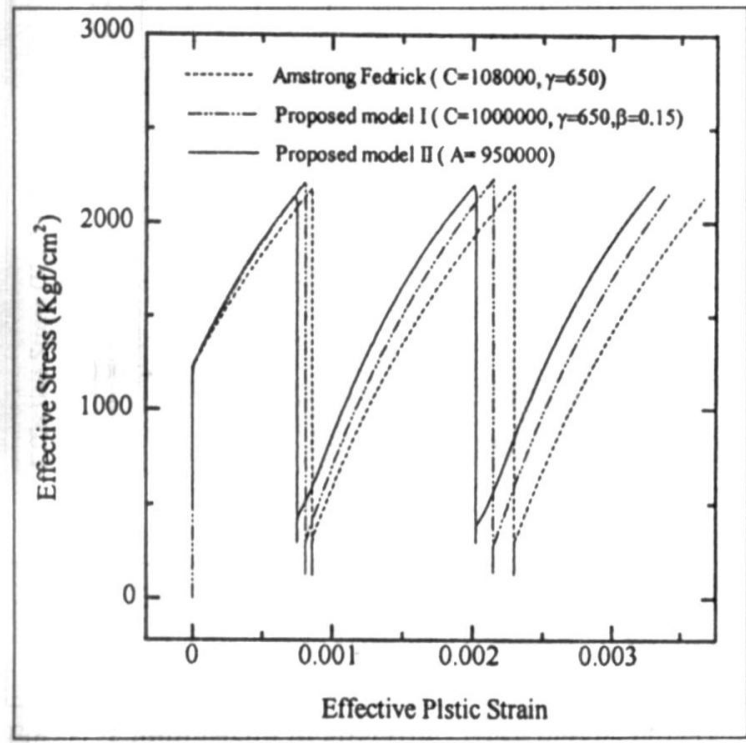

Fig. 11. Comparison of effective stress versus effective plastic strain behavior with previous models at highly stressed location.

The two numerical examples mentioned reveal that the contribution of proposed models provide more optimized prediction to the real application of cyclic elasto-plastic analysis and confirmes the significance of the proposed models in the civil engineering field.

\section{Case study}

\section{1 Considered bridge}

A case study was undertaken to estimate the remaining service life of an existing railway bridge by utilizing presented models to ensure the capability of application in real civil engineering structures. Since railway bridges are generally subjected to dynamic traffic loads, the present condition of an existing structure deviates from the original state with age and this causes unexpected stress concentrations at critical places. Low cycle fatigue is considered as the major criteria for failure at critical (highly stressed) location of the bridge. Consequently, the low cycle fatigue based remaining life estimation is presented here.

\section{2 Testing of bridge materials}

The bridge material was subjected to uniaxial tensile, reverse and cyclic loading operations following the ASTM E8 code and hence determined behaviour of wrought iron material is more close to kinematic hardening behaviour. The sample results are recorded to compare with the proposed fatigue models in chapter 6 (Fig. 4 \& 6).

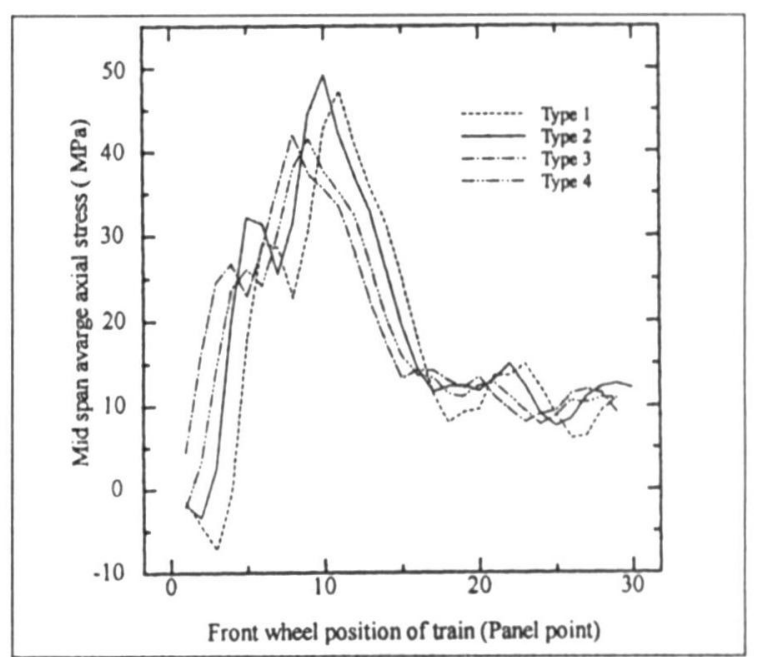

Fig. 12. Mid span average axial stress variation with front wheel position of moving train for diagonal members

\section{3 Stress evaluation and load testing on bridge}

The Bridge deck was analysed using the FE method employing the general-purpose package SAP 2000. Several member sets were used in the analysis of the structure based on various cross sectional properties. A 3D frame element model[17] of one complete middle span 
of the bridge was analyzed under test loadings and actual loadings to determine stresses in members and deflections under moving loads. The field static and dynamic load testing is based on site strain, displacement and acceleration measurements at various locations of the bridge. The static load test measurements demonstrate good agreement with FEM analytical results[17]. Hence, it concludes the validity of $\mathrm{FE}$ model and shows the possibility in other analyses involving actual loadings on the bridge

The field measurements express that the truss diagonal members are subjected to maximum range of stresses. The mid span (not close to the connection) axial stress variations with the train front wheel positions are plotted in Fig. 12 with respect to the measured strain. Since the truss diagonals also consist of four types of different cross sections, maximum strain consisting member behavior of each type is only deliberated to identify the critical member. Thus, it illustrates that the critical member is found from type 2 and it is subjected to around 350 to $500 \mathrm{Kgf} / \mathrm{cm} 2$ uniform tensile stress across mid span section due to variable amplitude actual loading.

Although the identified critical members with respective connections should be subjected to various non-destructive field tests which are used to detect the defects in highly stressed areas, the lack of facilities with experience hindered the quantitative investigation of the bridge. Hence, the remaining life estimation of the critical member is limited to the criteria called critical state of stress due to release of contactness of rivets.

\section{4 Life estimation}

To investigate the stress distribution around rivet holes, the most critical member of truss girder is subjected to further analysis. The ninenode isoperimetric shell elements were used for the FE mesh and it consists of 7490 nodes performing 1821 elements. To simulate the different unilateral contacts between rivet and plate, fully bonding, smooth contact and actual air gap restraint conditions are used.

Since the major objective of initial linear elastic analysis is to find out the most critical state of stresses around rivet holes, the different cases associated with different contact possibilities of the rivets with the plate are considered. Finally, it revealed that some locations in the connected members are subjected to multi axial stress state. Further, von Misses stress could exceed even the yield stress of the material, in some cases, and such cases are subjected to low cycle fatigue life estimation. Since the major criteria behind the life estimation is the determination of the effective plastic strain at critical location, the proposed fatigue model II based elasto-plastic analysis was performed. The von Misses stress distribution of the considerd three cases is as shown in Fig. 13 and it illustrates highly stressed locations clearly.

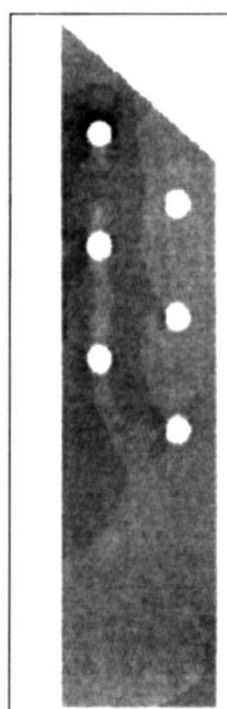

(a)

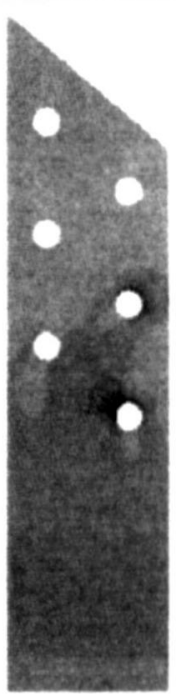

(b)

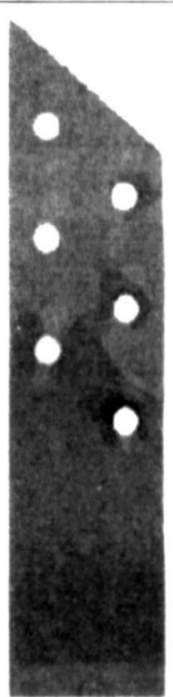

(c)
Fig. 13. Critical von Mises stress distribution for plastic loading (a). One active rivet is in contact (b). Two active rivets are in contact (c). Three active rivets are in contact. (The darkness shows the highly stressed location)

Since the critical member has never been subjected to compressive stress, the threshold value for the low cycle fatigue failure has resulted as a tensile failure strain of the wrought iron[17]. Time to reach the accumulated plastic strain to the failure strain of material is considered as the remaining fatigue life of the member. The sample diagram of effective stress versus effective plastic strain at the highly stressed locations of the member for single day traffic is plotted in Fig. 14 to discuss the behavior properly. 


\subsection{Results}

Out of various types of critical cases, it is revealed that the active number of rivets which are able to transfer the load deviates the fatigue life significantly. The summarized results are shown in Table 1.

Table 1: Predicted remaining fatigue life of the critical member in the bridge.

\begin{tabular}{|l|l|}
\hline Number of active rivets & Remaining fatigue life \\
\hline One rivet & 8 days \\
Two rivets & 4 months \\
Three rivets & 1 year \& 6 months \\
\hline
\end{tabular}

Even though the presented estimation is based on Fatigue model II, the prediction based on Fatigue model I also follows the same procedure in advance.

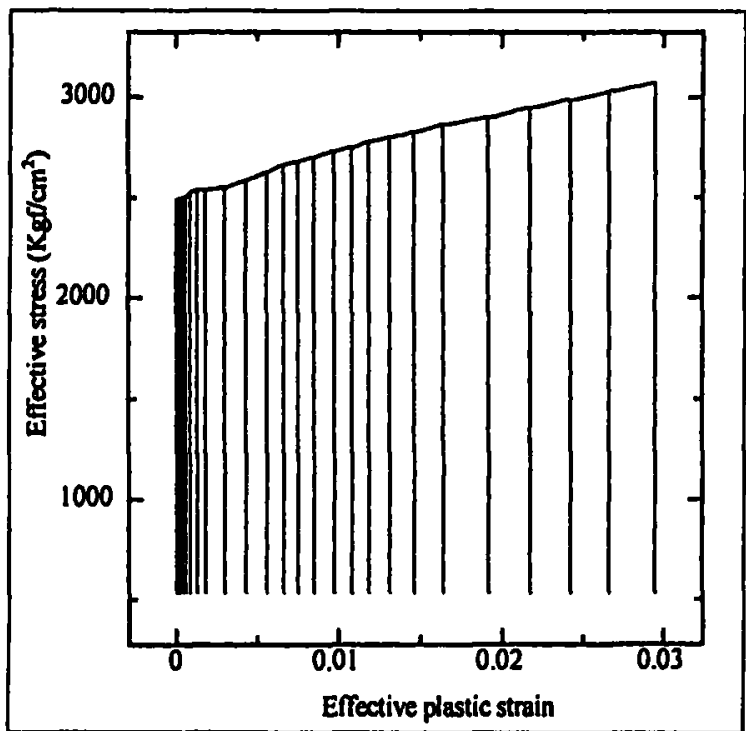

Fig. 14. Effective stress vs effective plastic strain behavior of the critical location when single rivet is in contact.

\section{Conclusions}

The results illustrate that the present model represents the real behavior of kinematic hardening material with higher accuracy than those of previous models in the vicinity of cyclic elasto-plastic analyses. Further, it is found that the contribution of the proposed model provides more optimized prediction to the real application of cyclic elasto-plastic analysis. Despite the two surfaces based proposed Fatigue model II is conceptually well accepted in the present day world, deviatoric stress and strain based numerical formulation arises to make it difficult in computational analysis. However, the modified Armstrong Fredrick based Fatigue model I produced relatively better correlation to real behaviour. The case study results illustrate that the present condition of places where the stresses are severe tends to deviate the fatigue life significantly. Therefore, in the frame of extraordinary inspection and maintenance of the bridge, it is of great importance to investigate accurately the condition of places where the stress concentration effect is severe such as notch, crack or connection area, especially in old bridges. Finally, it can be concluded that, when compared to previous low cycle fatigue techniques published in literature, these models based life estimations can be considered as one bounding approach which is not dependent on complex experiments.

Since this paper presents only the usage of uniaxail experimental results to verify the proposed models, the comparison with the biaxial experiments including more numerical examples under different loading scenarios are recommended for future studies.

\section{References}

(1) Jung, S. K. and Frangapol, D.M., Prediction of reliability and cost profiles of deteriorating bridges under time-and performance-controlled maintenance, Journal of Structural Engineering, ASCE, pp. 1865-1874, 2004.

(2) Fisher, J.W., Yen, B.T and Wang, D., Fatigue and fracture evaluation for rating riveted bridges, Washington National Cooperative Highwway Research Program report 302, National Research Council, USA, 1987.

(3) Shigley, J.E. and Mischke, C.R., Mechnnical Engineering Design, Fifth edition, New York, McGraw-Hill Book Company, 1989.

(4) Ranaweera, M.P.R., Aberuwan, H., Mauroof, A.L.M., Herath, K.R.B., Dissanayake, P.B.R., Siriwardane S.A.S.C. and Adasooriya, A.M.N.D., Structuml appraisal of railuay bridge at colombo over kelani river, Engineering Design Center, University of Peradeniya, Sri Lanka, 2002.

(5) Carden, L.P.,Itani, A.M. and Buckle, L.G., Seismic performance of steel girder bridges with ductile cross frames using single angle $X$ braces, Joumal of Structurnl Engineering, ASCE, pp. 329$337,2006$.

(6) Liu, W., Liang, Z. and George, C.L., Low-cycle bending-Fatigue strength of steel bars under random excitations. Part 1 \& II, Journal of Structural Engineering, ASCE, pp. 913-923, 2005. 
(7) Miki, C. and Sasaki, E., Fracture in steel bridge piers due to earthquakes, Internationnl Journal of Steel Structures, KSSC, pp. 133-140, 2005.

(8) Lee, G.C.,Liang, Z. and Ou, Y., Low-cycle fatigue in limit state seismic design of steel structures, 3rd International Symposium on Steel Structures, Seoul, Korea, 10-11 March, 2005.

(9) Navarro, A., Giraldez, J.M. and Vallellano, C., A constitutive model for elasto-plastic deformation under variable amplitude multiaxial cyclic loading, Internntional Joumal of Fatigue, Elsevier, pp. 838-846, 2005.

(10) Wang, C.H. and Brown, M.W., Life prediction techniques for variable amplitude multiaxial fatigue, Part 1 \& 2, Journal of Engineering Mnterinls and Teclinology, ASME, pp. 367-374, 1996.

(11) Frank, M., Narayanaswami, R., Jean, P. and Andre, B., A mesoscopic approach for fatigue life prediction under multiaxial loading, Multiaxinl Fatigue and Fracture, Opole, ESIS Publication 25, pp. 87-100, 1999.

(12) Endo, T., Damage Evaluation of Metals for random or varying loading-three aspect of the rainflow method, Proceedings of the 1974 Symposium on Mechanical Behaviour of Materials, Society of Material Science Japan, pp. 372-380, 1974.

(13) Hoffman, M. and Seegar, T., Local strain approach in multiaxial fatigue, Low Cycle Fatigue and Elasto-Plastic Behaviour of Mnterials, Elsevier, Applied Science Ltd., pp. 493-498, 1987.

(14) Wang, C.H. and Brown, M.W., In elastic deformation and fatigue under complex loading, Structural Mechanics in Reactor Technology, Elsevier, pp. 159-170, 1993.

(15) Park, J.S., Kim, S.J.,Kim, K.H.,Park, S.H. and Lee, C.S., A microstructural model for predicting high cycle fatigue, Internationnl Journal of Fatigue, Elsevier, pp. 1115-1123, 2005.

(16) Czyryca, E.J., Fatigue crack initiation, ASM Hand book, Fifth edition, pp. 365-375, 1995.

(17) Siriwardane, S.A.S.C., Fatigue life prediction of existing railway bridges, MPhil Thesis, University of Peradeniya, Sri Lanaka, 2004.

(18) Morel, F., A critical plane approach for life prediction of high cycle fatigue under multiaxial variable amplitude loading, International Journal of Fatigue, Elsevier, pp.101-119, 2000.

(19) Dafalias,Y.F. and Popov, E.P., Plastic internal variables formalism of cyclic plasticity, Journal of Applied Mechanics, ASME, 1976, pp. 645-651, 1976.

(20) Taher, M.A., and Voyiadjis, G.Z., Plastic damage model for concrete under cyclic multiaxial loading, Journal of Engineering Mechanics, pp. 1465-1484, 1993.
(21) Lubliner, J., Plasticity Theory, New York, Macmillan Publishing Company, $1990 .{ }^{\circ}$.

(22) Mroz, Z., On the description of anisotropic workhardening, Jourmal of the Mechanics and Physics of Solids, Elsevier, pp.163-175, 1967.

(23) Krieg, R.D., A practical two surface plasticity theory, Joumal of Applied Mechanics, ASME, pp. 641-646, 1975.

(24) Ohno, N. and Kachi, Y., A constitutive model of cyclic plasticity for nonlinear hardening materials, Journal of Applied Mechnnics, ASME, pp. 395-403, 1986.

(25) McDowell, D.L., A two surface model for transient nonproportional cyclic plasticity, Journal of Applied Mechanics, ASME, pp 298-302, 1985.

(26) Rashid, K.A.A., Material length scales in gradient-dependent plasticity/damage and size effect: Theory and computaion, P/DD thesis, Lousiana State University, U.S.A., 2004.

(27) Jiang, Y. and Kurath, P., Characteristics of the Armstrong- Fedrick type plasticity models, International Journnl of Plasticity, pp. 387-415, 1996.

(28) Gozzi, J., Practical behavior of steel (Experimental investigation and modeling), Licentinte Thesis, Lulea University of Technology, pp. 1402-1757, 2004.

(29) Lu, W.Y. and Mohamed, Z.M., A two-surafce plasticity theory and its application to multiaxial loading, Acta Mechanica 69, pp. 43-57, 1987.

(30) Hopperstad, O.S. and Remeseth, S., A return mapping algorithm for a class of cyclic plasticity models, International Journal for Numerical Methods in Engineering, John Wiley \& Sons, Ltd, pp. 549-564, 1995.

(31) Voyiajis, G.Z. and Basuroychowdhury, I.N., A plasticity model for multiaxial cyclic loading and ratcheting, Actn Meclinnica 126, pp. 19-35, 1998.

(32) Zienkiewicz, O.C., The Finite Elemet Method, 3rd Edition, McGraw-Hill book Company (UK) Limited, London, 1986. 